\title{
Approximation by bivariate generalized Bernstein-Schurer operators and associated GBS operators
}

\section{S.A. Mohiuddine $e^{1,2^{*}}$ (D)}

\section{"Correspondence:}

mohiuddine@gmail.com

'Department of General Required

Courses, Mathematics, Faculty of

Applied Studies, King Abdulaziz

University, Jeddah, 21589, Saudi

Arabia

${ }^{2}$ Operator Theory and Applications

Research Group, Department of

Mathematics, King Abdulaziz

University, Jeddah, 21589, Saudi

Arabia

\begin{abstract}
We construct the bivariate form of Bernstein-Schurer operators based on parameter $\alpha$. We establish the Voronovskaja-type theorem and give an estimate of the order of approximation with the help of Peetre's $K$-functional of our newly defined operators. Moreover, we define the associated generalized Boolean sum (shortly, GBS) operators and estimate the rate of convergence by means of mixed modulus of smoothness. Finally, the order of approximation for Bögel differentiable function of our GBS operators is presented.
\end{abstract}

MSC: 41A10; 41A25; 41A36

Keywords: $\alpha$-Bernstein operators; Bivariate $\alpha$-Bernstein-Schurer operators; GBS operators; Bögel differentiable function; Modulus of continuity

\section{Introduction}

Schurer [41] presented the modification of the classical Bernstein operators with the help of nonnegative parameter and nowadays called Bernstein-Schurer operators, which are linear and positive. Suppose that $\mathbb{Z}_{0}^{+}$and $C[a, b]$ are used to denote the space of nonnegative integers and continuous functions on $[a, b]$, respectively. Let us take $\eta \in \mathbb{Z}_{0}^{+}$. The well-known Bernstein-Schurer operators

$$
M_{j, \eta}: C[0,1+\eta] \longrightarrow C[0,1]
$$

are defined as

$$
M_{j, \eta}(g ; y)=\sum_{k=0}^{j+\eta} g\left(\frac{k}{j}\right) M_{j, \eta, k}(y)
$$

for any $g \in[0,1+\eta], j \in \mathbb{N}$, and $y \in[0,1]$, where

$$
M_{j, \eta, k}(y)=\left(\begin{array}{c}
j+\eta \\
k
\end{array}\right) y^{k}(1-y)^{j+\eta-k} .
$$

(c) The Author(s) 2020. This article is licensed under a Creative Commons Attribution 4.0 International License, which permits use, sharing, adaptation, distribution and reproduction in any medium or format, as long as you give appropriate credit to the original author(s) and the source, provide a link to the Creative Commons licence, and indicate if changes were made. The images or other third party material in this article are included in the article's Creative Commons licence, unless indicated otherwise in a credit line to the material. If material is not included in the article's Creative Commons licence and your intended use is not permitted by statutory regulation or exceeds the permitted use, you will need to obtain permission directly from the copyright holder. To view a copy of this licence, visit http://creativecommons.org/licenses/by/4.0/. 
When $\eta=0$ in (1.1), we obtain

$$
M_{j, 0}(g ; y)=\sum_{k=0}^{j} g\left(\frac{k}{j}\right) M_{j, 0, k}(y)=M_{j}(g ; y), \quad \text { say. }
$$

In this case, the operators $M_{j}(g ; y)$ and $M_{j, 0, k}(y)$, respectively, are called Bernstein operators and polynomials [11].

Most recently, the generalization of Bernstein operators was demonstrated by the authors Chen et al. [17] by taking the parameter $\alpha \in \mathbb{R}$. However, they showed that their operators are positive and linear for the choice of $0 \leq \alpha \leq 1$, so they considered this assumption in their work and then studied several approximation properties for their $\alpha$-Bernstein operators. Thereafter, many researchers, keeping the idea of this meaningful parameter $\alpha$ into account, constructed several operators. For example, Mohiuddine et al. [26] introduced the family of $\alpha$-Bernstein-Kantorovich operators and associated bivariate form and demonstrated the results regarding the rate of convergence via Peetre's $K$-functional together with modulus of continuity. In addition to this, the Stancu type $\alpha$-BernsteinKantorovich, $\alpha$-Baskakov and their Kantorovich form, and $\alpha$-Baskakov-Durrmeyer operators were analyzed by Mohiuddine and Özger [32], Aral et al. [6, 20], and Mohiuddine et al. [31], respectively, and for other blending type operators, see [23, 27, 36]. Some other modifications of Bernstein operators have been studied in [2, 15, 16, 25, 33, 34, 37, 42]. Furthermore, Acar and Kajla [3] gave the bivariate $\alpha$-Bernstein operators and associated generalized Boolean sum operators and then studied the degree of approximation of their operators. For more details on approximation by related operators and statistical approximation, we refer to $[1,4,10,14,21,28-30,35,40,44,45]$.

Motivated by the operators defined in (1.1) and $\alpha$-Bernstein operators, very recently, Ozger et al. [38] defined the $\alpha$-Bernstein-Schurer operators $M_{j, \eta}^{\alpha}: C[0,1+\eta] \longrightarrow C[0,1]$ by

$$
M_{j, \eta}^{\alpha}(g ; y)=\sum_{k=0}^{j+\eta} g_{k} M_{j, \eta, k}^{(\alpha)}(y)
$$

and

$$
g_{k}=g\left(\frac{k}{j}\right)
$$

for any $g \in C[0,1+\eta], y \in[0,1], j \in \mathbb{N}$, and $0 \leq \alpha \leq 1$, where

$$
M_{1, \eta, 0}^{(\alpha)}(y)=1-y, \quad M_{1, \eta, 1}^{(\alpha)}(y)=y
$$

and

$$
\begin{aligned}
M_{j, \eta, k}^{(\alpha)}(y)= & {\left[y(1-\alpha)\left(\begin{array}{c}
j+\eta-2 \\
k
\end{array}\right)+(1-y)(1-\alpha)\left(\begin{array}{c}
j+\eta-2 \\
k-2
\end{array}\right)\right.} \\
& \left.+y \alpha(1-y)\left(\begin{array}{c}
j+\eta \\
k
\end{array}\right)\right] y^{k-1}(1-y)^{j+\eta-k-1}
\end{aligned}
$$


for $j \geq 2$. Note that $M_{j, \eta}^{\alpha}(t-y ; y)=\eta y / j$. When $\eta=0$, the operators $M_{j, \eta}^{\alpha}(g ; y)$ coincide with $\alpha$-Bernstein operators. In addition to $\eta=0$, take $\alpha=1$, then $M_{j, \eta}^{\alpha}(g ; y)$ reduces to $M_{j}(g ; y)$. When only $\alpha=1$, the operators $M_{j, \eta}^{\alpha}(g ; y)$ reduce to the operators $M_{j, \eta}(g ; y)$. In the same paper, authors investigated global approximation, local approximation, and Voronovskajatype approximation results of the operators $M_{j, \eta}^{\alpha}(g ; y)$. They also established shape preserving properties such as monotonicity and convexity.

\section{Bivariate generalized Bernstein-Schurer operators}

Here, we construct the bivariate form of $\alpha$-Bernstein-Schurer operators and demonstrate their basic properties.

Throughout the manuscript, we suppose that $C\left(I^{2}\right)$ is the space of continuous function on $I^{2}(=I \times I)=[0,1+\eta] \times[0,1+\eta]$, where $\eta$ in $\mathbb{Z}_{0}^{+}$. For any $h \in C\left(I^{2}\right),\left(y_{1}, y_{2}\right) \in[0,1] \times[0,1]$, $s_{1}, s_{2} \in \mathbb{N}$, and $\alpha_{1}, \alpha_{2}$ in $[0,1]$, we define

$$
M_{s_{1}, s_{2}, \eta}^{\alpha_{1}, \alpha_{2}}\left(h ; y_{1}, y_{2}\right)=\sum_{\ell_{1}=0}^{s_{1}+\eta} \sum_{\ell_{2}=0}^{s_{2}+\eta} h\left(\frac{\ell_{1}}{s_{1}}, \frac{\ell_{2}}{s_{2}}\right) M_{s_{1}+\eta, s_{2}+\eta, \ell_{1}, \ell_{2}}^{\left(\alpha_{1}, \alpha_{2}\right)}\left(y_{1}, y_{2}\right),
$$

where the polynomials $M_{s_{1}+\eta, s_{2}+\eta, \ell_{1}, \ell_{2}}^{\left(\alpha_{1}, \alpha_{2}\right)}\left(y_{1}, y_{2}\right)=M_{s_{1}+\eta, \ell_{1}}^{\left(\alpha_{1}\right)}\left(y_{1}\right) M_{s_{2}+\eta, \ell_{2}}^{\left(\alpha_{2}\right)}\left(y_{2}\right)$ are considered by

$$
\begin{aligned}
M_{s_{1}+\eta, s_{2}+\eta, \ell_{1}, \ell_{2}}^{\left(\alpha_{1}, \alpha_{2}\right)}\left(y_{1}, y_{2}\right)= & {\left[\left(1-\alpha_{1}\right) y_{1}\left(\begin{array}{c}
s_{1}+\eta-2 \\
\ell_{1}
\end{array}\right)+\left(1-\alpha_{1}\right)\left(1-y_{1}\right)\left(\begin{array}{c}
s_{1}+\eta-2 \\
\ell_{1}-2
\end{array}\right)\right.} \\
& \left.+\alpha_{1} y_{1}\left(1-y_{1}\right)\left(\begin{array}{c}
s_{1}+\eta \\
\ell_{1}
\end{array}\right)\right] y_{1}^{\ell_{1}-1}\left(1-y_{1}\right)^{s_{1}+\eta-\left(\ell_{1}+1\right)} \\
& \times\left[\left(1-\alpha_{2}\right) y_{2}\left(\begin{array}{c}
s_{2}+\eta-2 \\
\ell_{2}
\end{array}\right)+\left(1-\alpha_{2}\right)\left(1-y_{2}\right)\left(\begin{array}{c}
s_{2}+\eta-2 \\
\ell_{2}-2
\end{array}\right)\right. \\
& \left.+\alpha_{2} y_{2}\left(1-y_{2}\right)\left(\begin{array}{c}
s_{2}+\eta \\
\ell_{2}
\end{array}\right)\right] y_{2}^{\ell_{2}-1}\left(1-y_{2}\right)^{s_{2}+\eta-\left(\ell_{2}+1\right)} .
\end{aligned}
$$

Lemma 2.1 Suppose that $e_{j k}\left(y_{1}, y_{2}\right)=y_{1}^{j} y_{2}^{k}$ for $(j, k)=\mathbb{N}_{0} \times \mathbb{N}_{0}\left(\mathbb{N}_{0}=\mathbb{N} \cup\{0\}\right)$ with $j+k \leq 4$.

Then

$$
\begin{aligned}
& M_{s_{1}, s_{2}, \eta}^{\alpha_{1}, \alpha_{2}}\left(e_{00} ; y_{1}, y_{2}\right)=1, \\
& M_{s_{1}, s_{2}, \eta}^{\alpha_{1}, \alpha_{2}}\left(e_{10} ; y_{1}, y_{2}\right)=\left(1+\frac{\eta}{s_{1}}\right) y_{1}, \\
& M_{s_{1}, s_{2}, \eta}^{\alpha_{1}, \alpha_{2}}\left(e_{01} ; y_{1}, y_{2}\right)=\left(1+\frac{\eta}{s_{2}}\right) y_{2}, \\
& M_{s_{1}, \alpha_{2}, \eta}^{\alpha_{1}, \alpha_{2}}\left(e_{20} ; y_{1}, y_{2}\right)=y_{1}^{2}+\frac{\left(s_{1}+\eta+2\left(1-\alpha_{1}\right)\right)\left(y_{1}-y_{1}^{2}\right)}{s_{1}^{2}}+\frac{\eta\left(\eta+2 s_{1}\right) y_{1}^{2}}{s_{1}^{2}}, \\
& M_{s_{1}, s_{2}, \eta}^{\alpha_{1}, \alpha_{2}}\left(e_{02} ; y_{1}, y_{2}\right)=y_{2}^{2}+\frac{\left(s_{2}+\eta+2\left(1-\alpha_{2}\right)\right)\left(y_{2}-y_{2}^{2}\right)}{s_{2}^{2}}+\frac{\eta\left(\eta+2 s_{2}\right) y_{2}^{2}}{s_{2}^{2}}, \\
& M_{s_{1}, s_{2}, \eta}^{\alpha_{1}, \alpha_{2}}\left(e_{30} ; y_{1}, y_{2}\right) \\
& \quad=y_{1}^{3}+\frac{s_{1}+\eta+6\left(1-\alpha_{1}\right)}{s_{1}^{3}} y_{1}+\left(-6 \alpha_{1} \eta-6 \alpha_{1} s_{1}+3 \eta^{2}+6 \eta s_{1}+3 s_{1}^{2}\right. \\
& \left.\quad+18 \alpha_{1}+3 \eta+3 s_{1}-18\right) \frac{y_{1}^{2}}{s_{1}^{3}}+\left(\eta^{3}+3 \eta^{2} s_{1}+3 \eta s_{1}^{2}+6 \alpha_{1} \eta+6 \alpha_{1} s_{1}\right.
\end{aligned}
$$




$$
\begin{aligned}
& \left.-3 \eta^{2}-6 \eta s_{1}-3 s_{1}^{2}-12 \alpha_{1}-4 \eta-4 s_{1}+12\right) \frac{y_{1}^{3}}{s_{1}^{3}} \\
& M_{s_{1}, s_{2}, \eta}^{\alpha_{1}, \alpha_{2}}\left(e_{03} ; y_{1}, y_{2}\right) \\
& =y_{2}^{3}+\frac{s_{2}+\eta+6\left(1-\alpha_{2}\right)}{s_{2}^{3}} y_{2}+\left(-6 \alpha_{2} \eta-6 \alpha_{2} s_{2}+3 \eta^{2}+6 \eta s_{2}+3 s_{2}^{2}\right. \\
& \left.+18 \alpha_{2}+3 \eta+3 s_{2}-18\right) \frac{y_{2}^{2}}{s_{2}^{3}}+\left(\eta^{3}+3 \eta^{2} s_{2}+3 \eta s_{2}^{2}+6 \alpha_{2} \eta+6 \alpha_{2} s_{2}\right. \\
& \left.-3 \eta^{2}-6 \eta s_{2}-3 s_{2}^{2}-12 \alpha_{2}-4 \eta-4 s_{2}+12\right) \frac{y_{2}^{3}}{s_{2}^{3}} \\
& M_{s_{1}, s_{2}, \eta}^{\alpha_{1}, \alpha_{2}}\left(e_{40} ; y_{1}, y_{2}\right) \\
& =y_{1}^{4}+\frac{s_{1}+\eta+14\left(1-\alpha_{1}\right)}{s_{1}^{4}} y_{1}+\left(-36 \alpha_{1} \eta-36 \alpha_{1} s_{1}+7 \eta^{2}+14 \eta s_{1}\right. \\
& \left.+7 s_{1}^{2}+86 \alpha_{1}+29 \eta+29 s_{1}-86\right) \frac{y_{1}^{2}}{s_{1}^{4}}+\left(-12 \alpha_{1} \eta^{2}-24 \alpha_{1} \eta s_{1}\right. \\
& -12 \alpha_{1} s_{1}^{2}+6 \eta^{3}+18 \eta^{2} s_{1}+18 \eta s_{1}^{2}+6 s_{1}^{3}+96 \alpha_{1} \eta+96 \alpha_{1} s_{1}-6 \eta^{2} \\
& \left.-12 \eta s_{1}-6 s_{1}^{2}-144 \alpha_{1}-84 \eta-84 s_{1}+144\right) \frac{y_{1}^{3}}{s_{1}^{4}}+\left(\eta^{4}+4 \eta^{3} s_{1}\right. \\
& +6 \eta^{2} s_{1}^{2}+4 \eta s_{1}^{3}+12 \alpha_{1} \eta^{2}+24 \alpha_{1} \eta s_{1}+12 \alpha_{1} s_{1}^{2}-6 \eta^{3}-18 \eta^{2} s_{1} \\
& -18 \eta s_{1}^{2}-6 s_{1}^{3}-60 \alpha_{1} \eta-60 \alpha_{1} s_{1}-\eta^{2}-2 \eta s_{1}-s_{1}^{2}+72 \alpha_{1}+54 \eta \\
& \left.+54 s_{1}-72\right) \frac{y_{1}^{4}}{s_{1}^{4}} \\
& M_{s_{1}, s_{2}, \eta}^{\alpha_{1}, \alpha_{2}}\left(e_{04} ; y_{1}, y_{2}\right) \\
& =y_{2}^{4}+\frac{s_{2}+\eta+14\left(1-\alpha_{2}\right)}{s_{2}^{4}} y_{2}+\left(-36 \alpha_{2} \eta-36 \alpha_{2} s_{2}+7 \eta^{2}+14 \eta s_{2}\right. \\
& \left.+7 s_{2}^{2}+86 \alpha_{2}+29 \eta+29 s_{2}-86\right) \frac{y_{2}^{2}}{s_{2}^{4}}+\left(-12 \alpha_{2} \eta^{2}-24 \alpha_{2} \eta s_{2}\right. \\
& -12 \alpha_{2} s_{2}^{2}+6 \eta^{3}+18 \eta^{2} s_{2}+18 \eta s_{2}^{2}+6 s_{2}^{3}+96 \alpha_{2} \eta+96 \alpha_{2} s_{2}-6 \eta^{2} \\
& \left.-12 \eta s_{2}-6 s_{2}^{2}-144 \alpha_{2}-84 \eta-84 s_{2}+144\right) \frac{y_{2}^{3}}{s_{2}^{4}}+\left(\eta^{4}+4 \eta^{3} s_{2}\right. \\
& +6 \eta^{2} s_{2}^{2}+4 \eta s_{2}^{3}+12 \alpha_{2} \eta^{2}+24 \alpha_{2} \eta s_{2}+12 \alpha_{2} s_{2}^{2}-6 \eta^{3}-18 \eta^{2} s_{2} \\
& -18 \eta s_{2}^{2}-6 s_{2}^{3}-60 \alpha_{2} \eta-60 \alpha_{2} s_{2}-\eta^{2}-2 \eta s_{2}-s_{2}^{2}+72 \alpha_{2}+54 \eta \\
& \left.+54 s_{2}-72\right) \frac{y_{2}^{4}}{s_{2}^{4}}
\end{aligned}
$$

Proof We shall use Lemma 3 of [38] to prove Lemma 2.1. Clearly, from (2.1), we obtain

$$
M_{s_{1}, s_{2}, \eta}^{\alpha_{1}, \alpha_{2}}\left(e_{00} ; y_{1}, y_{2}\right)=\sum_{\ell_{1}=0}^{s_{1}+\eta} \sum_{\ell_{2}=0}^{s_{2}+\eta} M_{s_{1}+\eta, s_{2}+\eta, \ell_{1}, \ell_{2}}^{\left(\alpha_{1}, \alpha_{2}\right)}\left(y_{1}, y_{2}\right)=1
$$


Next, we write

$$
\begin{aligned}
M_{s_{1}, s_{2}, \eta}^{\alpha_{1}, \alpha_{2}}\left(e_{10} ; y_{1}, y_{2}\right) & =\sum_{\ell_{1}=0}^{s_{1}+\eta} \sum_{\ell_{2}=0}^{s_{2}+\eta} \frac{\ell_{1}}{s_{1}} M_{s_{1}+\eta, s_{2}+\eta, \ell_{1}, \ell_{2}}^{\left(\alpha_{1}, \alpha_{2}\right)}\left(y_{1}, y_{2}\right) \\
& =\sum_{\ell_{1}=0}^{s_{1}+\eta} \frac{\ell_{1}}{s_{1}} M_{s_{1}+\eta, \ell_{1}}^{\left(\alpha_{1}\right)}\left(y_{1}\right) \sum_{\ell_{2}=0}^{s_{2}+\eta} M_{s_{2}+\eta, \ell_{2}}^{\left(\alpha_{2}\right)}\left(y_{2}\right) \\
& =M_{s_{1}, \eta}^{\alpha}\left(e_{1} ; y_{1}\right) M_{s_{2}, \eta}^{\alpha}\left(e_{0} ; y_{2}\right) \\
& =\left(1+\frac{\eta}{s_{1}}\right) y_{1} .
\end{aligned}
$$

Similarly, we get

$$
M_{s_{1}, s_{2}, \eta}^{\alpha_{1}, \alpha_{2}}\left(e_{01} ; y_{1}, y_{2}\right)=M_{s_{1}, \eta}^{\alpha}\left(e_{0} ; y_{1}\right) M_{s_{2}, \eta}^{\alpha}\left(e_{1} ; y_{2}\right)=\left(1+\frac{\eta}{s_{2}}\right) y_{2}
$$

Further,

$$
\begin{aligned}
M_{s_{1}, s_{2}, \eta}^{\alpha_{1}, \alpha_{2}}\left(e_{20} ; y_{1}, y_{2}\right) & =\sum_{\ell_{1}=0}^{s_{1}+\eta}\left(\frac{\ell_{1}}{s_{1}}\right)^{2} M_{s_{1}+\eta, \ell_{1}}^{\left(\alpha_{1}\right)}\left(y_{1}\right) \sum_{\ell_{2}=0}^{s_{2}+\eta} M_{s_{2}+\eta, \ell_{2}}^{\left(\alpha_{2}\right)}\left(y_{2}\right) \\
& =M_{s_{1}, \eta}^{\alpha}\left(e_{2} ; y_{1}\right) M_{s_{2}, \eta}^{\alpha}\left(e_{0} ; y_{2}\right) \\
& =y_{1}^{2}+\frac{\left(s_{1}+\eta+2\left(1-\alpha_{1}\right)\right)\left(y_{1}-y_{1}^{2}\right)}{s_{1}^{2}}+\frac{\eta\left(\eta+2 s_{1}\right) y_{1}^{2}}{s_{1}^{2}},
\end{aligned}
$$

and similarly, we obtain

$$
\begin{aligned}
M_{s_{1}, s_{2}, \eta}^{\alpha_{1}, \alpha_{2}}\left(e_{02} ; y_{1}, y_{2}\right) & =\sum_{\ell_{1}=0}^{s_{1}+\eta} M_{s_{1}+\eta, \ell_{1}}^{\left(\alpha_{1}\right)}\left(y_{1}\right) \sum_{\ell_{2}=0}^{s_{2}+\eta}\left(\frac{\ell_{2}}{s_{2}}\right)^{2} M_{s_{2}+\eta, \ell_{2}}^{\left(\alpha_{2}\right)}\left(y_{2}\right) \\
& =M_{s_{1}, \eta}^{\alpha}\left(e_{0} ; y_{1}\right) M_{s_{2}, \eta}^{\alpha}\left(e_{2} ; y_{2}\right) \\
& =y_{2}^{2}+\frac{\left(s_{2}+\eta+2\left(1-\alpha_{2}\right)\right)\left(y_{2}-y_{2}^{2}\right)}{s_{2}^{2}}+\frac{\eta\left(\eta+2 s_{2}\right) y_{2}^{2}}{s_{2}^{2}} .
\end{aligned}
$$

Similarly, we obtain the last two moments.

\section{Corollary 2.2 The following identities hold:}

$$
\begin{aligned}
& M_{s_{1}, s_{2}, \eta}^{\alpha_{1}, \alpha_{2}}\left(t_{1}-y_{1} ; y_{1}, y_{2}\right)=\frac{\eta y_{1}}{s_{1}}, \\
& M_{s_{1}, s_{2}, \eta}^{\alpha_{1}, \alpha_{2}}\left(t_{2}-y_{2} ; y_{1}, y_{2}\right)=\frac{\eta y_{2}}{s_{2}}, \\
& M_{s_{1}, s_{2}, \eta}^{\alpha_{1}, \alpha_{2}}\left(\left(t_{1}-y_{1}\right)^{2} ; y_{1}, y_{2}\right)=\frac{\left(s_{1}+\eta+2\left(1-\alpha_{1}\right)\right)\left(y_{1}-y_{1}^{2}\right)+\eta^{2} y_{1}^{2}}{s_{1}^{2}}, \\
& M_{s_{1}, s_{2}, \eta}^{\alpha_{1}, \alpha_{2}}\left(\left(t_{2}-y_{2}\right)^{2} ; y_{1}, y_{2}\right)=\frac{\left(s_{2}+\eta+2\left(1-\alpha_{2}\right)\right)\left(y_{2}-y_{2}^{2}\right)+\eta^{2} y_{2}^{2}}{s_{2}^{2}}, \\
& M_{s_{1}, s_{2}, \eta}^{\alpha_{1}, \alpha_{2}}\left(\left(t_{1}-y_{1}\right)^{4} ; y_{1}, y_{2}\right)
\end{aligned}
$$




$$
\begin{aligned}
& =\left(s_{1}+\eta+14\left(1-\alpha_{1}\right)\right) \frac{y_{1}}{s_{1}^{4}}+\left(-36 \alpha_{1} \eta-12 \alpha_{1} s_{1}+7 \eta^{2}\right. \\
& \left.+10 \eta s_{1}+3 s_{1}^{2}+86 \alpha_{1}+29 \eta+5 s_{1}-86\right) \frac{y_{1}^{2}}{s_{1}^{4}}+\left(-12 \alpha_{1} \eta^{2}\right. \\
& +6 \eta^{3}+6 \eta^{2} s_{1}+96 \alpha_{1} \eta+24 \alpha_{1} s_{1}-6 \eta^{2}-24 \eta s_{1}-6 s_{1}^{2} \\
& \left.-144 \alpha_{1}-84 \eta-12 s_{1}+144\right) \frac{y_{1}^{3}}{s_{1}^{4}}+\left(\eta^{4}+12 \alpha_{1} \eta^{2}-6 \eta^{3}\right. \\
& -6 \eta^{2} s_{1}-60 \alpha_{1} \eta-12 \alpha_{1} s_{1}-\eta^{2}+14 \eta s_{1}+3 s_{1}^{2}+72 \alpha_{1} \\
& \left.+54 \eta+6 s_{1}-72\right) \frac{y_{1}^{4}}{s_{1}^{4}} \\
& M_{s_{1}, s_{2}, \eta}^{\alpha_{1}, \alpha_{2}}\left(\left(t_{2}-y_{2}\right)^{4} ; y_{1}, y_{2}\right) \\
& =\left(s_{2}+\eta+14\left(1-\alpha_{2}\right)\right) \frac{y_{2}}{s_{2}^{4}}+\left(-36 \alpha_{2} \eta-12 \alpha_{2} s_{2}+7 \eta^{2}\right. \\
& \left.+10 \eta s_{2}+3 s_{2}^{2}+86 \alpha_{2}+29 \eta+5 s_{2}-86\right) \frac{y_{2}^{2}}{s_{2}^{4}}+\left(-12 \alpha_{2} \eta^{2}\right. \\
& +6 \eta^{3}+6 \eta^{2} s_{2}+96 \alpha_{2} \eta+24 \alpha_{2} s_{2}-6 \eta^{2}-24 \eta s_{2}-6 s_{2}^{2} \\
& \left.-144 \alpha_{2}-84 \eta-12 s_{2}+144\right) \frac{y_{2}^{3}}{s_{2}^{4}}+\left(\eta^{4}+12 \alpha_{2} \eta^{2}-6 \eta^{3}\right. \\
& -6 \eta^{2} s_{2}-60 \alpha_{2} \eta-12 \alpha_{2} s_{2}-\eta^{2}+14 \eta s_{2}+3 s_{2}^{2}+72 \alpha_{2} \\
& \left.+54 \eta+6 s_{2}-72\right) \frac{y_{2}^{4}}{s_{2}^{4}}
\end{aligned}
$$

\section{Order of convergence and Voronovskaja-type results}

In this section, we obtain the Voronovskaja-type result and the order of convergence with the help of Peetre's $K$-functional for our operators $M_{s_{1}, s_{2}, \eta}^{\alpha_{1}, \alpha_{2}}\left(h ; y_{1}, y_{2}\right)$. For $g \in C\left(I^{2}\right)$, the norm of the bivariate function $g$ is considered by

$$
\|g\|_{C\left(I^{2}\right)}=\sup _{\left(y_{1}, y_{2}\right) \in \mathcal{I}^{2}}\left|g\left(y_{1}, y_{2}\right)\right| .
$$

Theorem 3.1 For any $h \in C\left(I^{2}\right)$, one has

$$
\lim _{s_{1}, s_{2} \rightarrow \infty}\left\|M_{s_{1}, s_{2}, \eta}^{\alpha_{1}, \alpha_{2}}(h)-h\right\|_{C\left(I_{1}^{2}\right)}=0
$$

where $I_{1}^{2}=[0,1] \times[0,1]$.

Proof We see that

$$
\begin{array}{ll}
\left\|M_{s_{1}, s_{2}, \eta}^{\alpha_{1}, \alpha_{2}}\left(e_{00}\right)-e_{00}\right\|_{C\left(I_{1}^{2}\right)} \rightarrow 0, & \left\|M_{s_{1}, s_{2}, \eta}^{\alpha_{1}, \alpha_{2}}\left(e_{10}\right)-e_{10}\right\|_{C\left(I_{1}^{2}\right)} \rightarrow 0, \\
\left\|M_{s_{1}, s_{2}, \eta}^{\alpha_{1}, \alpha_{2}}\left(e_{01}\right)-e_{01}\right\|_{C\left(I_{1}^{2}\right)} \rightarrow 0, & \left\|M_{s_{1}, s_{2}, \eta}^{\alpha_{1}, \alpha_{2}}\left(e_{20}+e_{02}\right)-\left(e_{20}+e_{02}\right)\right\|_{C\left(I_{1}^{2}\right)} \rightarrow 0
\end{array}
$$

as $s_{1}, s_{2} \rightarrow \infty$. Thus (3.1) holds by Volkov's theorem [43]. 
We will use $C^{2}\left(I^{2}\right)$ to denote the space of all functions $h \in C\left(I^{2}\right)$ such that $\frac{\partial^{j} h}{\partial y_{1}^{j}}, \frac{\partial^{j} h}{\partial y_{2}^{j}}, \frac{\partial^{2} h}{\partial y_{1} \partial y_{2}} \in$ $C\left(I^{2}\right)(j=1,2)$ and equipped with the norm

$$
\|h\|_{C^{2}\left(I^{2}\right)}=\|h\|_{C\left(I^{2}\right)}+\sum_{j=1}^{2}\left(\left\|\frac{\partial^{j} h}{\partial y_{1}^{j}}\right\|_{C\left(I^{2}\right)}+\left\|\frac{\partial^{j} h}{\partial y_{2}^{j}}\right\|_{C\left(I^{2}\right)}\right)+\left\|\frac{\partial^{2} h}{\partial y_{1} \partial y_{2}}\right\|_{C\left(I^{2}\right)} \quad\left(h \in C\left(I^{2}\right)\right) .
$$

Theorem 3.2 Suppose that $h \in C^{2}\left(I^{2}\right)$. Then

$$
\begin{aligned}
\lim _{s \rightarrow \infty} s\left(M_{s, s, \eta}^{\alpha_{1}, \alpha_{2}}\left(h ; y_{1}, y_{2}\right)-h\left(y_{1}, y_{2}\right)\right) \\
=\eta\left(y_{1} h_{y_{1}}\left(y_{1}, y_{2}\right)+y_{2} h_{y_{2}}\left(y_{1}, y_{2}\right)\right) \\
\quad+\frac{y_{1}\left(1-y_{1}\right)}{2} h_{y_{1}, y_{1}}\left(y_{1}, y_{2}\right)+\frac{y_{2}\left(1-y_{2}\right)}{2} h_{y_{2}, y_{2}}\left(y_{1}, y_{2}\right)
\end{aligned}
$$

uniformly on $I_{1}^{2}$.

Proof Suppose that $h \in C^{2}\left(I^{2}\right)$. Then Taylor's theorem gives

$$
\begin{aligned}
h\left(t_{1}, t_{2}\right)= & h\left(y_{1}, y_{2}\right)+h_{y_{1}}\left(y_{1}, y_{2}\right)\left(t_{1}-y_{1}\right)+h_{y_{2}}\left(y_{1}, y_{2}\right)\left(t_{2}-y_{2}\right) \\
& +\frac{1}{2}\left[h_{y_{1} y_{1}}\left(y_{1}, y_{2}\right)\left(t_{1}-y_{1}\right)^{2}+2 h_{y_{1} y_{2}}\left(y_{1}, y_{2}\right)\left(t_{1}-y_{1}\right)\left(t_{2}-y_{2}\right)\right. \\
& \left.+h_{y_{2} y_{2}}\left(y_{1}, y_{2}\right)\left(t_{2}-y_{2}\right)^{2}\right]+\rho\left(t_{1}, t_{2}, y_{1}, y_{2}\right) \sqrt{\left(t_{1}-y_{1}\right)^{4}+\left(t_{2}-y_{2}\right)^{4}},
\end{aligned}
$$

where $\rho\left(t_{1}, t_{2}, y_{1}, y_{2}\right) \in C\left(I^{2}\right)$ and

$$
\rho\left(t_{1}, t_{2}, y_{1}, y_{2}\right) \rightarrow 0\left(\left(t_{1}, t_{2}\right) \rightarrow\left(y_{1}, y_{2}\right)\right) .
$$

Since $M_{s, s, \eta}^{\alpha_{1}, \alpha_{2}}$ is linear so, by operating on (3.2), we obtain

$$
\begin{aligned}
M_{s, s, \eta}^{\alpha_{1}, \alpha_{2}} & \left(h\left(t_{1}, t_{2}\right) ; y_{1}, y_{2}\right) \\
= & h\left(y_{1}, y_{2}\right)+h_{y_{1}}\left(y_{1}, y_{2}\right) M_{s, s, \eta}^{\alpha_{1}, \alpha_{2}}\left(t_{1}-y_{1} ; y_{1}, y_{2}\right) \\
& +h_{y_{2}}\left(y_{1}, y_{2}\right) M_{s, s, \eta}^{\alpha_{1}, \alpha_{2}}\left(t_{2}-y_{2} ; y_{1}, y_{2}\right) \\
& +\frac{1}{2}\left[h_{y_{1} y_{1}}\left(y_{1}, y_{2}\right) M_{s, s, \eta}^{\alpha_{1}, \alpha_{2}}\left(\left(t_{1}-y_{1}\right)^{2} ; y_{1}, y_{2}\right)\right. \\
& +2 h_{y_{1} y_{2}}\left(y_{1}, y_{2}\right) M_{s, s, \eta}^{\alpha_{1}, \alpha_{2}}\left(\left(t_{1}-y_{1}\right)\left(t_{2}-y_{2}\right) ; y_{1}, y_{2}\right) \\
& \left.\left.+h_{y_{2} y_{2}}\left(y_{1}, y_{2}\right) M_{s, s, \eta}^{\alpha_{1}, \alpha_{2}}\left(\left(t_{2}-y_{2}\right)^{2} ; y_{1}, y_{2}\right)\right)\right] \\
& +M_{s, s, \eta}^{\alpha_{1}, \alpha_{2}}\left(\rho\left(t_{1}, t_{2}, y_{1}, y_{2}\right) \sqrt{\left(t_{1}-y_{1}\right)^{4}+\left(t_{2}-y_{2}\right)^{4}} ; y_{1}, y_{2}\right) .
\end{aligned}
$$

With a view of Corollary 2.2, we find that

$$
\begin{aligned}
\lim _{s \rightarrow \infty} s M_{s, s, \eta}^{\alpha_{1}, \alpha_{2}}\left(\left(t_{1}-y_{1}\right)\left(t_{2}-y_{2}\right) ; y_{1}, y_{2}\right)= & \lim _{s \rightarrow \infty} s\left[M_{s, \eta}^{\alpha_{1}}\left(t_{1}-y_{1} ; y_{1}\right)\right. \\
& \left.\times M_{s, \eta}^{\alpha_{2}}\left(t_{2}-y_{2} ; y_{2}\right)\right] \\
= & 0
\end{aligned}
$$


and also

$$
\begin{aligned}
& \lim _{s \rightarrow \infty} s M_{s, s, \eta}^{\alpha_{1}, \alpha_{2}}\left(t_{1}-y_{1} ; y_{1}, y_{2}\right)=\eta y_{1}, \quad \lim _{s \rightarrow \infty} s M_{s, s, \eta}^{\alpha_{1}, \alpha_{2}}\left(t_{2}-y_{2} ; y_{1}, y_{2}\right)=\eta y_{2}, \\
& \lim _{s \rightarrow \infty} s M_{s, s, \eta}^{\alpha_{1}, \alpha_{2}}\left(\left(t_{1}-y_{1}\right)^{2} ; y_{1}, y_{2}\right)=y_{1}\left(1-y_{1}\right), \\
& \lim _{s \rightarrow \infty} s M_{s, s, \eta}^{\alpha_{1}, \alpha_{2}}\left(\left(t_{2}-y_{2}\right)^{2} ; y_{1}, y_{2}\right)=y_{2}\left(1-y_{2}\right) .
\end{aligned}
$$

It follows from (3.3)-(3.6) that

$$
\begin{aligned}
\lim _{s \rightarrow \infty} & s\left(M_{s, s, \eta}^{\alpha_{1}, \alpha_{2}}\left(h ; y_{1}, y_{2}\right)-h\left(y_{1}, y_{2}\right)\right) \\
= & \eta y_{1} h_{y_{1}}\left(y_{1}, y_{2}\right)+\eta y_{2} h_{y_{2}}\left(y_{1}, y_{2}\right) \\
& +\frac{1}{2}\left[y_{1}\left(1-y_{1}\right) h_{y_{1} y_{1}}\left(y_{1}, y_{2}\right)+y_{2}\left(1-y_{2}\right) h_{y_{2} y_{2}}\left(y_{1}, y_{2}\right)\right] \\
& +\lim _{s \rightarrow \infty} s M_{s, s, \eta}^{\alpha_{1}, \alpha_{2}}\left(\rho\left(t_{1}, t_{2}, y_{1}, y_{2}\right) \sqrt{\left(t_{1}-y_{1}\right)^{4}+\left(t_{2}-y_{2}\right)^{4}} ; y_{1}, y_{2}\right) .
\end{aligned}
$$

By the Cauchy-Schwarz inequality, one gets

$$
\begin{aligned}
& s M_{s, s, \eta}^{\alpha_{1}, \alpha_{2}}\left(\rho\left(t_{1}, t_{2}, y_{1}, y_{2}\right) \sqrt{\left(t_{1}-y_{1}\right)^{4}+\left(t_{2}-y_{2}\right)^{4}} ; y_{1}, y_{2}\right) \\
& \leq \sqrt{M_{s, s, \eta}^{\alpha_{1}, \alpha_{2}}\left(\rho^{2}\left(t_{1}, t_{2}, y_{1}, y_{2}\right) ; y_{1}, y_{2}\right)} \sqrt{s^{2} M_{s, s, \eta}^{\alpha_{1}, \alpha_{2}}\left(\left(t_{1}-y_{1}\right)^{4}+\left(t_{2}-y_{2}\right)^{4} ; y_{1}, y_{2}\right)} \\
&= \sqrt{M_{s, s, \eta}^{\alpha_{1}, \alpha_{2}}\left(\rho^{2}\left(t_{1}, t_{2}, y_{1}, y_{2}\right) ; y_{1}, y_{2}\right)} \\
& \times \sqrt{s^{2}\left(M_{s, s, \eta}^{\alpha_{1}, \alpha_{2}}\left(\left(t_{1}-y_{1}\right)^{4} ; y_{1}, y_{2}\right)+M_{s, s, \eta}^{\alpha_{1}, \alpha_{2}}\left(\left(t_{2}-y_{2}\right)^{4} ; y_{1}, y_{2}\right)\right)} .
\end{aligned}
$$

Since $\rho\left(t_{1}, t_{2}, y_{1}, y_{2}\right) \in C\left(I^{2}\right)$ and $\lim _{\left(t_{1}, t_{2}\right) \rightarrow\left(y_{1}, y_{2}\right)} \rho\left(t_{1}, t_{2}, y_{1}, y_{2}\right)=0$, we have

$$
\lim _{s \rightarrow \infty} M_{s, s, \eta}^{\alpha_{1}, \alpha_{2}}\left(\rho^{2}\left(t_{1}, t_{2}, y_{1}, y_{2}\right) ; y_{1}, y_{2}\right)=0
$$

uniformly on $I_{1}^{2}$ by Theorem 3.1. Corollary 2.2 gives

$$
s^{2} M_{s, s, \eta}^{\alpha_{1}, \alpha_{2}}\left(\left(t_{1}-y_{1}\right)^{4} ; y_{1}, y_{2}\right) \longrightarrow 3 y_{1}^{2}-6 y_{1}^{3}+3 y_{1}^{4} \quad(s \longrightarrow \infty)
$$

and

$$
s^{2} M_{s, s, \eta}^{\alpha_{1}, \alpha_{2}}\left(\left(t_{2}-y_{2}\right)^{4} ; y_{1}, y_{2}\right) \longrightarrow 3 y_{2}^{2}-6 y_{2}^{3}+3 y_{2}^{4} \quad(s \longrightarrow \infty) .
$$

Employing the last three relations in Eq. (3.8) and then using Eq. (3.7), we obtain

$$
\begin{array}{rl}
\lim _{s \rightarrow \infty} s & s\left(M_{s, s, \eta}^{\alpha_{1}, \alpha_{2}}\left(h ; y_{1}, y_{2}\right)-h\left(y_{1}, y_{2}\right)\right) \\
= & \eta y_{1} h_{y_{1}}\left(y_{1}, y_{2}\right)+\eta y_{2} h_{y_{2}}\left(y_{1}, y_{2}\right) \\
& +\frac{1}{2}\left[y_{1}\left(1-y_{1}\right) h_{y_{1} y_{1}}\left(y_{1}, y_{2}\right)+y_{2}\left(1-y_{2}\right) h_{y_{2} y_{2}}\left(y_{1}, y_{2}\right)\right]
\end{array}
$$

uniformly on $I_{1}^{2}$. 
For $h \in C\left(I^{2}\right)$ and $\delta>0$, Peetre's $K$-functional is given by

$$
K(h ; \delta)=\inf _{h_{1} \in C^{2}\left(I^{2}\right)}\left\{\left\|h-h_{1}\right\|_{C\left(I^{2}\right)}+\delta\left\|h_{1}\right\|_{C\left(I^{2}\right)}\right\}
$$

and the modulus of continuity of $h$ is

$$
\omega(h ; \delta)=\sup \left\{\left|h\left(u_{1}, u_{2}\right)-h\left(y_{1}, y_{2}\right)\right|:\left(u_{1}, u_{2}\right),\left(y_{1}, y_{2}\right) \in I_{1}^{2}, \sqrt{\left(u_{1}-y_{1}\right)^{2}+\left(u_{2}-y_{2}\right)^{2}} \leq \delta\right\} .
$$

By Theorem 9 (see [18]), there is a constant $C>0$ such that

$$
K(h ; \delta) \leq C \omega_{2}(h ; \sqrt{\delta}) .
$$

In the above relation, $\omega_{2}(h ; \sqrt{\delta})$ is the second-order modulus of continuity of $h \in C\left(I^{2}\right)$ (for details, see [5]).

Theorem 3.3 For any $h \in C\left(I^{2}\right)$, one has

$$
\left|M_{s_{1}, s_{2}, \eta}^{\alpha_{1}, \alpha_{2}}\left(h ; y_{1}, y_{2}\right)-h\left(y_{1}, y_{2}\right)\right| \leq 4 K\left(h ; \delta_{s_{1}, s_{2}}\left(y_{1}, y_{2}\right)\right)+\omega\left(h ; \sqrt{\left(\frac{\eta y_{1}}{s_{1}}\right)^{2}+\left(\frac{\eta y_{2}}{s_{2}}\right)^{2}}\right)
$$

where

$$
\delta_{s_{1}, s_{2}}\left(y_{1}, y_{2}\right)(=\delta)=\frac{1}{4}\left(\delta_{s_{1}}^{2}\left(y_{1}\right)+\delta_{s_{2}}^{2}\left(y_{2}\right)+\left(\frac{\eta y_{1}}{s_{1}}\right)^{2}+\left(\frac{\eta y_{2}}{s_{2}}\right)^{2}\right)>0
$$

and

$$
\delta_{s_{1}}^{2}\left(y_{1}\right)=\mathcal{M}_{s_{1}, s_{2}, \eta}^{\alpha_{1}, \alpha_{2}}\left(\left(t_{1}-y_{1}\right)^{2} ; y_{1}, y_{2}\right), \quad \delta_{s_{2}}^{2}\left(y_{2}\right)=\mathcal{M}_{s_{1}, s_{2}, \eta}^{\alpha_{1}, \alpha_{2}}\left(\left(t_{2}-y_{2}\right)^{2} ; y_{1}, y_{2}\right) .
$$

Proof Assume that $h_{1} \in C^{2}\left(I^{2}\right)$. Then, by Taylor's theorem, we have

$$
\begin{aligned}
& h_{1}\left(t_{1}, t_{2}\right)-h_{1}\left(y_{1}, y_{2}\right) \\
& =\frac{\partial h_{1}\left(y_{1}, y_{2}\right)}{\partial y_{1}}\left(t_{1}-y_{1}\right)+\int_{y_{1}}^{t_{1}}\left(t_{1}-u_{1}\right) \frac{\partial^{2} h_{1}\left(u_{1}, y_{2}\right)}{\partial u_{1}^{2}} d u_{1} \\
& \quad+\frac{\partial h_{1}\left(y_{1}, y_{2}\right)}{\partial y_{2}}\left(t_{2}-y_{2}\right)+\int_{y_{2}}^{t_{2}}\left(t_{2}-u_{2}\right) \frac{\partial^{2} h_{1}\left(y_{1}, u_{2}\right)}{\partial u_{2}^{2}} d u_{2} .
\end{aligned}
$$

We are now defining the auxiliary operators by

$$
\mathcal{M}_{s_{1}, s_{2}, \eta}^{\alpha_{1}, \alpha_{2}}\left(h ; y_{1}, y_{2}\right)=M_{s_{1}, s_{2}, \eta}^{\alpha_{1}, \alpha_{2}}\left(h ; y_{1}, y_{2}\right)-h\left(\frac{\left(\eta+s_{1}\right) y_{1}}{s_{1}}, \frac{\left(\eta+s_{2}\right) y_{2}}{s_{2}}\right)+h\left(y_{1}, y_{2}\right)
$$

Simple calculation together with Corollary 2.2 gives that

$$
\mathcal{M}_{s_{1}, s_{2}, \eta}^{\alpha_{1}, \alpha_{2}}\left(t_{1}-y_{1} ; y_{1}, y_{2}\right)=0 \quad \text { and } \quad \mathcal{M}_{s_{1}, s_{2}, \eta}^{\alpha_{1}, \alpha_{2}}\left(t_{2}-y_{2} ; y_{1}, y_{2}\right)=0
$$


By operating $\mathcal{M}_{s_{1}, s_{2}, \eta}^{\alpha_{1}, \alpha_{2}}$ in (3.10) and using the last relation, we obtain

$$
\begin{aligned}
& \mathcal{M}_{s_{1}, s_{2}, \eta}^{\alpha_{1}, \alpha_{2}}\left(h_{1} ; y_{1}, y_{2}\right)-h_{1}\left(y_{1}, y_{2}\right) \\
&=\mathcal{M}_{s_{1}, s_{2}, \eta}^{\alpha_{1}, \alpha_{2}}\left(\int_{y_{1}}^{t_{1}}\left(t_{1}-u_{1}\right) \frac{\partial^{2} h_{1}\left(u_{1}, y_{2}\right)}{\partial u_{1}^{2}} d u_{1} ; y_{1}, y_{2}\right) \\
&+\mathcal{M}_{s_{1}, s_{2}, \eta}^{\alpha_{1}, \alpha_{2}}\left(\int_{y_{2}}^{t_{2}}\left(t_{2}-u_{2}\right) \frac{\partial^{2} h_{1}\left(y_{1}, u_{2}\right)}{\partial u_{2}^{2}} d u_{2} ; y_{1}, y_{2}\right) \\
&= M_{s_{1}, s_{2}, \eta}^{\alpha_{1}, \alpha_{2}}\left(\int_{y_{1}}^{t_{1}}\left(t_{1}-u_{1}\right) \frac{\partial^{2} h_{1}\left(u_{1}, y_{2}\right)}{\partial u_{1}^{2}} d u_{1} ; y_{1}, y_{2}\right) \\
&-\left(\int_{y_{1}}^{\frac{\left(\eta+s_{1}\right) y_{1}}{s_{1}}}\left(\frac{\left(\eta+s_{1}\right) y_{1}}{s_{1}}-u_{1}\right) \frac{\partial^{2} h_{1}\left(u_{1}, y_{2}\right)}{\partial u_{1}^{2}} d u_{1} ; y_{1}, y_{2}\right) \\
&+M_{s_{1}, s_{2}, \eta}^{\alpha_{1}, \alpha_{2}}\left(\int_{y_{2}}^{t_{2}}\left(t_{2}-u_{2}\right) \frac{\partial^{2} h_{1}\left(y_{1}, u_{2}\right)}{\partial u_{2}^{2}} d u_{2} ; y_{1}, y_{2}\right) \\
&-\left(\int_{y_{2}}^{\frac{\left(\eta+s_{2}\right) y_{2}}{s_{2}}}\left(\frac{\left(\eta+s_{2}\right) y_{2}}{s_{2}}-u_{2}\right) \frac{\partial^{2} h_{1}\left(y_{1}, u_{2}\right)}{\partial u_{2}^{2}} d u_{2} ; y_{1}, y_{2}\right),
\end{aligned}
$$

which yields

$$
\begin{aligned}
\left|\mathcal{M}_{s_{1}, s_{2}, \eta}^{\alpha_{1}, \alpha_{2}}\left(h_{1} ; y_{1}, y_{2}\right)-h_{1}\left(y_{1}, y_{2}\right)\right| & \leq \\
\leq & \left(M_{s_{1}, s_{2}, \eta}^{\alpha_{1}, \alpha_{2}}\left(\left(t_{1}-y_{1}\right)^{2} ; y_{1}, y_{2}\right)+\left(\frac{\eta y_{1}}{s_{1}}\right)^{2}\right)\left\|h_{1}\right\|_{C^{2}\left(I^{2}\right)} \\
& +\left(M_{s_{1}, s_{2}, \eta}^{\alpha_{1}, \alpha_{2}}\left(\left(t_{2}-y_{2}\right)^{2} ; y_{1}, y_{2}\right)+\left(\frac{\eta y_{2}}{s_{2}}\right)^{2}\right)\left\|h_{1}\right\|_{C^{2}\left(I^{2}\right)} \\
= & \left(\delta_{s_{1}}^{2}\left(y_{1}\right)+\delta_{s_{2}}^{2}\left(y_{2}\right)+\left(\frac{\eta y_{1}}{s_{1}}\right)^{2}+\left(\frac{\eta y_{2}}{s_{2}}\right)^{2}\right)\left\|h_{1}\right\|_{C^{2}\left(I^{2}\right)} .
\end{aligned}
$$

We can see that

$$
\left|\mathcal{M}_{s_{1}, s_{2}, \eta}^{\alpha_{1}, \alpha_{2}}\left(h ; y_{1}, y_{2}\right)\right| \leq\left|M_{s_{1}, s_{2}, \eta}^{\alpha_{1}, \alpha_{2}}\left(h ; y_{1}, y_{2}\right)\right|+\left|h\left(\frac{\left(\eta+s_{1}\right) y_{1}}{s_{1}}, \frac{\left(\eta+s_{2}\right) y_{2}}{s_{2}}\right)\right|+\left|h\left(y_{1}, y_{2}\right)\right|,
$$

which yields $\left|\mathcal{M}_{s_{1}, s_{2}, \eta}^{\alpha_{1}, \alpha_{2}}\left(h ; y_{1}, y_{2}\right)\right| \leq 3\|h\|$ for any $h \in C\left(I^{2}\right)$. We therefore write

$$
\begin{aligned}
& \left|M_{s_{1}, s_{2}, \eta}^{\alpha_{1}, \alpha_{2}}\left(h ; y_{1}, y_{2}\right)-h\left(y_{1}, y_{2}\right)\right| \\
& \leq\left|\mathcal{M}_{s_{1}, s_{2}, \eta}^{\alpha_{1}, \eta}\left(h-h_{1} ; y_{1}, y_{2}\right)\right|+\left|h\left(y_{1}, y_{2}\right)-h_{1}\left(y_{1}, y_{2}\right)\right| \\
& \quad+\left|\mathcal{M}_{s_{1}, s_{2}, \eta}^{\alpha_{1}, \alpha_{2}}\left(h_{1} ; y_{1}, y_{2}\right)-h_{1}\left(y_{1}, y_{2}\right)\right| \\
& \quad+\left|h\left(\frac{\left(\eta+s_{1}\right) y_{1}}{s_{1}}, \frac{\left(\eta+s_{2}\right) y_{2}}{s_{2}}\right)-h\left(y_{1}, y_{2}\right)\right| \\
& \leq 4\left\|h-h_{1}\right\|+\left(\delta_{s_{1}}^{2}\left(y_{1}\right)+\delta_{s_{2}}^{2}\left(y_{2}\right)+\left(\frac{\eta y_{1}}{s_{1}}\right)^{2}+\left(\frac{\eta y_{2}}{s_{2}}\right)^{2}\right)\left\|h_{1}\right\|_{C^{2}\left(I^{2}\right)} \\
& \quad+\omega\left(h ; \sqrt{\left(\frac{\eta y_{1}}{s_{1}}\right)^{2}+\left(\frac{\eta y_{2}}{s_{2}}\right)^{2}}\right) .
\end{aligned}
$$


Now, taking $\inf _{h_{1} \in C^{2}\left(I^{2}\right)}$, we get

$$
\left|M_{s_{1}, s_{2}, \eta}^{\alpha_{1}, \alpha_{2}}\left(h ; y_{1}, y_{2}\right)-h\left(y_{1}, y_{2}\right)\right| \leq 4 K\left(h ; \delta_{s_{1}, s_{2}}\left(y_{1}, y_{2}\right)\right)+\omega\left(h ; \sqrt{\left(\frac{\eta y_{1}}{s_{1}}\right)^{2}+\left(\frac{\eta y_{2}}{s_{2}}\right)^{2}}\right)
$$

which completes the proof.

The following corollary follows from Theorem 3.3 and inequality (3.9).

Corollary 3.4 Let $h \in C\left(I^{2}\right)$. Then

$$
\begin{aligned}
& \left|M_{s_{1}, s_{2}, \eta}^{\alpha_{1}, \alpha_{2}}\left(h ; y_{1}, y_{2}\right)-h\left(y_{1}, y_{2}\right)\right| \\
& \quad \leq C \omega_{2}\left(h ; \sqrt{\delta_{s_{1}, s_{2}}\left(y_{1}, y_{2}\right)}\right)+\omega\left(h ; \sqrt{\left(\frac{\eta y_{1}}{s_{1}}\right)^{2}+\left(\frac{\eta y_{2}}{s_{2}}\right)^{2}}\right) .
\end{aligned}
$$

\section{GBS operators of bivariate generalized Bernstein-Schurer type}

For any compact real intervals $X$ and $Y$, the function $h: Y_{1} \times Y_{2} \rightarrow \mathbb{R}$ is $B$-bounded (or Bögel bounded) on $Y_{1} \times Y_{2}$ if there exists $H>0$ such that

$$
\left|\Delta_{\left(y_{1}, y_{2}\right)} h\left[u_{1}, u_{2} ; y_{1}, y_{2}\right]\right| \leq H \quad\left(\forall\left(u_{1}, u_{2}\right),\left(y_{1}, y_{2}\right) \in Y_{1} \times Y_{2}\right),
$$

where $\Delta_{\left(y_{1}, y_{2}\right)} h\left[u_{1}, u_{2} ; y_{1}, y_{2}\right]$ is the mixed difference of $h$ defined by

$$
\Delta_{\left(y_{1}, y_{2}\right)} h\left[u_{1}, u_{2} ; y_{1}, y_{2}\right]=h\left(y_{1}, y_{2}\right)-h\left(y_{1}, u_{2}\right)-h\left(u_{1}, y_{2}\right)+h\left(u_{1}, u_{2}\right) .
$$

A function $h$ is said to be $B$-continuous (or Bögel continuous) at a point $\left(u_{1}, u_{2}\right)$ if

$$
\lim _{\left(y_{1}, y_{2}\right) \rightarrow\left(u_{1}, u_{2}\right)} \Delta_{\left(y_{1}, y_{2}\right)} h\left[u_{1}, u_{2} ; y_{1}, y_{2}\right]=0
$$

for any $\left(u_{1}, u_{2}\right),\left(y_{1}, y_{2}\right) \in Y_{1} \times Y_{2}$ (see [13]).

Given a function $h \in C\left(I^{2}\right)$, for any $s_{1}, s_{2} \in \mathbb{N}, \eta \in \mathbb{Z}_{0}^{+}$, and $\alpha_{1}, \alpha_{2} \in[0,1]$, we define the generalized Boolean sum (or GBS) operators of the bivariate form of generalized Bernstein-Schurer operators (2.1) by

$$
\Phi_{s_{1}, s_{2}, \eta}^{\alpha_{1}, \alpha_{2}}\left(h ; y_{1}, y_{2}\right)=M_{s_{1}, s_{2}, \eta}^{\alpha_{1}, \alpha_{2}}\left(h\left(t_{1}, y_{2}\right)+h\left(y_{1}, t_{2}\right)-h\left(t_{1}, t_{2}\right) ; y_{1}, y_{2}\right), \quad\left(\left(y_{1}, y_{2}\right) \in I_{1}^{2}\right),
$$

or, equivalently, we write

$$
\begin{aligned}
\Phi_{s_{1}, s_{2}, \eta}^{\alpha_{1}, \alpha_{2}}\left(h ; y_{1}, y_{2}\right)= & \sum_{\ell_{1}=0}^{s_{1}+\eta} \sum_{\ell_{2}=0}^{s_{2}+\eta} M_{s_{1}+\eta, s_{2}+\eta, \ell_{1}, \ell_{2}}^{\left(\alpha_{1}, \alpha_{2}\right)}\left(y_{1}, y_{2}\right) \\
& \times\left(h\left(\frac{\ell_{1}}{s_{1}}, y_{2}\right)+h\left(y_{1}, \frac{\ell_{2}}{s_{2}}\right)-h\left(\frac{\ell_{1}}{s_{1}}, \frac{\ell_{2}}{s_{2}}\right)\right) .
\end{aligned}
$$

Note that $\Phi_{s_{1}, s_{2}, \eta}^{\alpha_{1}, \alpha_{2}}\left(h ; y_{1}, y_{2}\right)$ is well defined on $C_{B}\left(I^{2}\right)$ (the space of all $B$-continuous functions on $\left.I^{2}\right)$ into $C\left(I^{2}\right)$ and $h \in C_{B}\left(I^{2}\right)$ as well as linear and positive. 
Recall that the mixed modulus of smoothness of $h \in C_{B}\left(I^{2}\right)$ is given by

$$
\begin{aligned}
\omega_{\text {mixed }}\left(h ; \delta_{1}, \delta_{2}\right)= & \sup \left\{\left|\Delta_{\left(y_{1}, y_{2}\right)} h\left[t_{1}, t_{2} ; y_{1}, y_{2}\right]\right|:\left|y_{1}-t_{1}\right|<\delta_{1},\left|y_{2}-t_{2}\right|<\delta_{2} ;\right. \\
& \left.\left(y_{1}, y_{2}\right),\left(u_{1}, u_{2}\right) \in I^{2}\right\}
\end{aligned}
$$

for any $\delta_{1}, \delta_{2}>0($ see $[7,9])$.

A function $h$ is $B$-differentiable (or Bögel differentiable) at the point $\left(u_{1}, u_{2}\right) \in Y_{1} \times Y_{2}$ if

$$
\lim _{\left(y_{1}, y_{2}\right) \rightarrow\left(u_{1}, u_{2}\right)} \frac{\Delta_{\left(y_{1}, y_{2}\right)} f\left[u_{1}, u_{2} ; y_{1}, y_{2}\right]}{\left(y_{1}-u_{1}\right)\left(y_{2}-u_{2}\right)}
$$

exists. The limit is called $B$-differentiable of $h$ at $\left(u_{1}, u_{2}\right)$ and denoted by $D_{y_{1} y_{2}} h\left(u_{1}, u_{2}\right)=$ $D_{B}\left(h ; u_{1}, u_{2}\right)$. By $D_{b}\left(Y_{1} \times Y_{2}\right)$, we denote the set of all $B$-differentiable functions.

For more details and related results, we refer to [8, 12, 19, 22, 24, 39].

The following theorem gives an estimate of the rate of convergence of $\Phi_{s_{1}, s_{2}, \eta}^{\alpha_{1}, \alpha_{2}}$ to $h \in$ $C_{B}\left(I^{2}\right)$.

Theorem 4.1 For any $h \in C_{B}\left(I^{2}\right)$, the inequality

$$
\left|\Phi_{s_{1}, s_{2}, \eta}^{\alpha_{1}, \alpha_{2}}\left(h ; y_{1}, y_{2}\right)-h\left(y_{1}, y_{2}\right)\right| \leq 4 \omega_{\text {mixed }}\left(h ; \sqrt{\beta_{s_{1}, \eta}}, \sqrt{\beta_{s_{2}, \eta}}\right)
$$

holds, where

$$
\beta_{s_{1}, \eta}=\frac{3+\eta(1+\eta)}{s_{1}} \quad \text { and } \quad \beta_{s_{2}, \eta}=\frac{3+\eta(1+\eta)}{s_{2}}
$$

Proof It follows from (4.3) and

$$
\omega_{\text {mixed }}\left(h ; \lambda_{2} \delta_{1}, \lambda_{2} \delta_{2}\right) \leq\left(1+\lambda_{1}\right)\left(1+\lambda_{2}\right) \omega_{\text {mixed }}\left(h ; \delta_{1}, \delta_{2}\right) \quad\left(\lambda_{1}, \lambda_{1}>0\right)
$$

that

$$
\begin{aligned}
\left|\Delta_{\left(y_{1}, y_{2}\right)} h\left[t_{1}, t_{2} ; y_{1}, y_{2}\right]\right| & \leq \omega_{\text {mixed }}\left(h ;\left|t_{1}-y_{1}\right|,\left|t_{2}-y_{2}\right|\right) \\
& \leq\left(1+\frac{\left|t_{1}-y_{1}\right|}{\delta_{1}}\right)\left(1+\frac{\left|t_{2}-y_{2}\right|}{\delta_{2}}\right) \omega_{\text {mixed }}\left(h ; \delta_{1}, \delta_{2}\right)
\end{aligned}
$$

for all $\left(y_{1}, y_{2}\right),\left(t_{1}, t_{2}\right) \in I^{2}$ and for any $\delta_{1}, \delta_{2}>0$. Rewrite (4.1) as

$$
h\left(y_{1}, t_{2}\right)+h\left(t_{1}, y_{2}\right)-h\left(t_{1}, t_{2}\right)=h\left(y_{1}, y_{2}\right)-\Delta_{\left(y_{1}, y_{2}\right)} h\left[t_{1}, t_{2} ; y_{1}, y_{2}\right]
$$

Operating $M_{s_{1}, s_{2}, \eta}^{\alpha_{1}, \alpha_{2}}$ and using the definition of $\Phi_{s_{1}, s_{2}, \eta}^{\alpha_{1}, \alpha_{2}}$, we obtain

$$
\Phi_{s_{1}, s_{2}, \eta}^{\alpha_{1}, \alpha_{2}}\left(h ; y_{1}, y_{2}\right)=h\left(y_{1}, y_{2}\right) M_{s_{1}, s_{2}, \eta}^{\alpha_{1}, \alpha_{2}}\left(e_{00} ; y_{1}, y_{2}\right)-M_{s_{1}, s_{2}, \eta}^{\alpha_{1}, \alpha_{2}}\left(\Delta_{\left(y_{1}, y_{2}\right)} h\left[t_{1}, t_{2} ; y_{1}, y_{2}\right] ; y_{1}, y_{2}\right)
$$

which yields

$$
\left|\Phi_{s_{1}, s_{2}, \eta}^{\alpha_{1}, \alpha_{2}}\left(h ; y_{1}, y_{2}\right)-h\left(y_{1}, y_{2}\right)\right| \leq M_{s_{1}, s_{2}, \eta}^{\alpha_{1}, \alpha_{2}}\left(\left|\Delta_{\left(y_{1}, y_{2}\right)} h\left[t_{1}, t_{2} ; y_{1}, y_{2}\right]\right| ; y_{1}, y_{2}\right)
$$


Employing (4.4) and then using the Cauchy-Schwarz inequality, we get

$$
\begin{aligned}
&\left|\Phi_{s_{1}, s_{2}, \eta}^{\alpha_{1}, \alpha_{2}}\left(h ; y_{1}, y_{2}\right)-h\left(y_{1}, y_{2}\right)\right| \\
& \leq\left(M_{s_{1}, s_{2}, \eta}^{\alpha_{1}, \alpha_{2}}\left(e_{00} ; y_{1}, y_{2}\right)+\delta_{1}^{-1} \sqrt{M_{s_{1}, s_{2}, \eta}^{\alpha_{1}, \alpha_{2}}\left(\left(t_{1}-y_{1}\right)^{2} ; y_{1}, y_{2}\right)}\right. \\
&+\delta_{2}^{-1} \sqrt{M_{s_{1}, s_{2}, \eta}^{\alpha_{1}, \alpha_{2}}\left(\left(t_{2}-y_{2}\right)^{2} ; y_{1}, y_{2}\right)} \\
&\left.+\delta_{1}^{-1} \delta_{2}^{-1} \sqrt{M_{s_{1}, s_{2}, \eta}^{\alpha_{1}, \alpha_{2}}\left(\left(t_{1}-y_{1}\right)^{2} ; y_{1}, y_{2}\right) M_{s_{1}, s_{2}, \eta}^{\alpha_{1}, \alpha_{2}}\left(\left(t_{2}-y_{2}\right)^{2} ; y_{1}, y_{2}\right)}\right) \omega_{\text {mixed }}\left(h ; \delta_{1}, \delta_{2}\right) .
\end{aligned}
$$

Using Corollary 2.2, we write

$$
\begin{aligned}
M_{s_{1}, s_{2}, \eta}^{\alpha_{1}, \alpha_{2}}\left(\left(t_{1}-y_{1}\right)^{2} ; y_{1}, y_{2}\right) & \leq \frac{y_{1}\left(1-y_{1}\right)}{s_{1}}+\frac{y_{1}\left(1-y_{1}\right)(\eta+2)}{s_{1}^{2}}+\frac{\eta^{2} y_{1}^{2}}{s_{1}^{2}} \\
& \leq \frac{3+\eta(1+\eta)}{s_{1}}=\beta_{s_{1}, \eta}
\end{aligned}
$$

and

$$
M_{s_{1}, s_{2}, \eta}^{\alpha_{1}, \alpha_{2}}\left(\left(t_{2}-y_{2}\right)^{2} ; y_{1}, y_{2}\right) \leq \frac{3+\eta(1+\eta)}{s_{2}}=\beta_{s_{2}, \eta} .
$$

We therefore obtain

$$
\begin{aligned}
\left|\Phi_{s_{1}, s_{2}, \eta}^{\alpha_{1}, \alpha_{2}}\left(h ; y_{1}, y_{2}\right)-h\left(y_{1}, y_{2}\right)\right| \leq & \left(1+\frac{1}{\delta_{1}} \sqrt{\frac{3+\eta(1+\eta)}{s_{1}}}\right. \\
& +\frac{1}{\delta_{1} \delta_{2}} \sqrt{\frac{3+\eta(1+\eta)}{s_{1}}} \sqrt{\frac{3+\eta(1+\eta)}{s_{2}}} \\
& \left.+\frac{1}{\delta_{2}} \sqrt{\frac{3+\eta(1+\eta)}{s_{2}}}\right) \omega_{\text {mixed }}\left(h ; \delta_{1}, \delta_{2}\right),
\end{aligned}
$$

which gives the assertion of Theorem 4.1 by choosing $\delta_{1}=\sqrt{\beta_{s_{1}, \eta}}$ and $\delta_{2}=\sqrt{\beta_{s_{2}, \eta}}$.

Finally, we study the order of approximation for $B$-differentiable functions of our operators $\Phi_{s_{1}, s_{2}, \eta}^{\alpha_{1}, \alpha_{2}}$.

Theorem 4.2 Suppose that $h \in D_{b}\left(I^{2}\right)$ and $D_{B} h$ in $B\left(I^{2}\right)$ (the space of all bounded functions on $\left.I^{2}\right)$. Then

$$
\left|\Phi_{s_{1}, s_{2}, \eta}^{\alpha_{1}, \alpha_{2}}\left(h ; y_{1}, y_{2}\right)-h\left(y_{1}, y_{2}\right)\right| \leq \frac{N}{\sqrt{s_{1} s_{2}}}\left\{\omega_{\text {mixed }}\left(D_{B} h ; \sqrt{\frac{1}{s_{1}}}, \sqrt{\frac{1}{s_{2}}}\right)+\left\|D_{B} h\right\|_{\infty}\right\},
$$

where $N>0$ is a constant.

Proof Let $h \in D_{b}\left(I^{2}\right)$. Then, from (see [13], p. 62), we write

$$
\Delta_{\left(y_{1}, y_{2}\right)} h\left[t_{1}, t_{2} ; y_{1}, y_{2}\right]=\left(t_{1}-y_{1}\right)\left(t_{2}-y_{2}\right) D_{B} h(\alpha, \gamma)
$$


for $y_{1}<\alpha<t_{1}, y_{2}<\gamma<t_{2}$. Thus, we fairly have

$$
D_{B} h(\alpha, \gamma)=\Delta_{\left(y_{1}, y_{2}\right)} D_{B} h(\alpha, \gamma)+D_{B} h\left(\alpha, y_{2}\right)+D_{B} h\left(y_{1}, \gamma\right)-D_{B} h\left(y_{1}, y_{2}\right) .
$$

Since $D_{B} h \in B\left(I^{2}\right)$, we have $\left|D_{B} h\left(y_{1}, y_{2}\right)\right| \leq\left\|D_{B} h\right\|_{\infty}$. In view of the last two equalities, we obtain

$$
\begin{aligned}
&\left|M_{s_{1}, s_{2}, \eta}^{\alpha_{1}, \alpha_{2}}\left(\Delta_{\left(y_{1}, y_{2}\right)} h\left[t_{1}, t_{2} ; y_{1}, y_{2}\right] ; y_{1}, y_{2}\right)\right| \\
&=\left|M_{s_{1}, s_{2}, \eta}^{\alpha_{1}, \alpha_{2}}\left(\left(t_{1}-y_{1}\right)\left(t_{2}-y_{2}\right) D_{B} h(\alpha, \gamma) ; y_{1}, y_{2}\right)\right| \\
& \leq M_{s_{1}, s_{2}, \eta}^{\alpha_{1}, \alpha_{2}}\left(\left|t_{1}-y_{1}\right|\left|t_{2}-y_{2}\right|\left|\Delta_{y_{1}, y_{2}} D_{B} h(\alpha, \gamma)\right| ; y_{1}, y_{2}\right) \\
&+M_{s_{1}, s_{2}, \eta}^{\alpha_{1}, \alpha_{2}}\left(| t _ { 1 } - y _ { 1 } | | t _ { 2 } - y _ { 2 } | \left(\left|D_{B} h\left(\alpha, y_{2}\right)\right|+\left|D_{B} h\left(y_{1}, \gamma\right)\right|\right.\right. \\
&\left.\left.+\left|D_{B} h\left(y_{1}, y_{2}\right)\right|\right) ; y_{1}, y_{2}\right) \\
& \leq M_{s_{1}, s_{2}, \eta}^{\alpha_{1}, \alpha_{2}}\left(\left|t_{1}-y_{1}\right|\left|t_{2}-y_{2}\right| \omega_{\text {mixed }}\left(D_{B} h ;\left|\alpha-y_{1}\right|,\left|\gamma-y_{2}\right|\right) ; y_{1}, y_{2}\right) \\
&+3\left\|D_{B} h\right\|_{\infty} M_{s_{1}, s_{2}, \eta}^{\alpha_{1}, \alpha_{2}}\left(\left|t_{1}-y_{1}\right|\left|t_{2}-y_{2}\right| ; y_{1}, y_{2}\right) .
\end{aligned}
$$

Also, we have

$$
\begin{aligned}
\omega_{\text {mixed }}\left(D_{B} h ;\left|\alpha-y_{1}\right|,\left|\gamma-y_{2}\right|\right) & \leq \omega_{\text {mixed }}\left(D_{B} h ;\left|t_{1}-y_{1}\right|,\left|t_{2}-y_{2}\right|\right) \\
& \leq\left(1+\frac{\left|t_{1}-y_{1}\right|}{\delta_{1}}\right)\left(1+\frac{\left|t_{2}-y_{2}\right|}{\delta_{2}}\right) \omega_{\text {mixed }}\left(h ; \delta_{1}, \delta_{2}\right) .
\end{aligned}
$$

We thus have from (4.5) and (4.6) together with the Cauchy-Schwarz inequality that

$$
\begin{aligned}
&\left|\Phi_{s_{1}, s_{2}, \eta}^{\alpha_{1}, \alpha_{2}}\left(h ; y_{1}, y_{2}\right)-h\left(y_{1}, y_{2}\right)\right| \\
&=\mid\left|M_{s_{1}, s_{2}, \eta}^{\alpha_{1}, \alpha_{2}}\left(\Delta_{\left(y_{1}, y_{2}\right)} h\left[t_{1}, t_{2} ; y_{1}, y_{2}\right] ; y_{1}, y_{2}\right)\right| \\
& \leq\left\{M_{s_{1}, s_{2}, \eta}^{\alpha_{1}, \alpha_{2}}\left(\left|t_{1}-y_{1}\right|\left|t_{2}-y_{2}\right| ; y_{1}, y_{2}\right)\right. \\
&+\delta_{1}^{-1} M_{s_{1}, s_{2}, \eta}^{\alpha_{1}, \alpha_{2}}\left(\left(t_{1}-y_{1}\right)^{2}\left|t_{2}-y_{2}\right| ; y_{1}, y_{2}\right) \\
&+\delta_{2}^{-1} M_{s_{1}, s_{2}, \eta}^{\alpha_{1}, \alpha_{2}}\left(\left|t_{1}-y_{1}\right|\left(t_{2}-y_{2}\right)^{2} ; y_{1}, y_{2}\right) \\
&\left.+\delta_{1}^{-1} \delta_{2}^{-1} M_{s_{1}, s_{2}, \eta}^{\alpha_{1}, \alpha_{2}}\left(\left(t_{1}-y_{1}\right)^{2}\left(t_{2}-y_{2}\right)^{2} ; y_{1}, y_{2}\right)\right\} \\
& \times \omega_{\text {mixed }}\left(D_{B} h ; \delta_{1}, \delta_{2}\right) \\
&+3\left\|D_{B} h\right\|_{\infty} \sqrt{M_{s_{1}, s_{2}, \eta}^{\alpha_{1}, \alpha_{2}}\left(\left(t_{1}-y_{1}\right)^{2}\left(t_{2}-y_{2}\right)^{2} ; y_{1}, y_{2}\right)},
\end{aligned}
$$

which gives

$$
\begin{aligned}
& \left|\Phi_{s_{1}, s_{2}, \eta}^{\alpha_{1}, \alpha_{2}}\left(h ; y_{1}, y_{2}\right)-h\left(y_{1}, y_{2}\right)\right| \\
& \leq\left\{\sqrt{M_{s_{1}, s_{2}, \eta}^{\alpha_{1}, \alpha_{2}}\left(\left(t_{1}-y_{1}\right)^{2}\left(t_{2}-y_{2}\right)^{2} ; y_{1}, y_{2}\right)}\right. \\
& \quad+\delta_{1}^{-1} \sqrt{M_{s_{1}, s_{2}, \eta}^{\alpha_{1}, \alpha_{2}}\left(\left(t_{1}-y_{1}\right)^{4}\left(t_{2}-y_{2}\right)^{2} ; y_{1}, y_{2}\right)} \\
& \quad+\delta_{2}^{-1} \sqrt{M_{s_{1}, s_{2}, \eta}^{\alpha_{1}, \alpha_{2}}\left(\left(t_{1}-y_{1}\right)^{2}\left(t_{2}-y_{2}\right)^{4} ; y_{1}, y_{2}\right)}
\end{aligned}
$$




$$
\begin{aligned}
& \left.+\delta_{1}^{-1} \delta_{2}^{-1} M_{s_{1}, s_{2}, \eta}^{\alpha_{1}, \alpha_{2}}\left(\left(t_{1}-y_{1}\right)^{2}\left(t_{2}-y_{2}\right)^{2} ; y_{1}, y_{2}\right)\right\} \\
& \times \omega_{\text {mixed }}\left(D_{B} h ; \delta_{1}, \delta_{2}\right) \\
& +3\left\|D_{B} h\right\|_{\infty} \sqrt{M_{s_{1}, s_{2}, \eta}^{\alpha_{1}, \alpha_{2}}\left(\left(t_{1}-y_{1}\right)^{2}\left(t_{2}-y_{2}\right)^{2} ; y_{1}, y_{2}\right)} .
\end{aligned}
$$

By straightforward calculation (from Corollary 2.2), we obtain

$$
\begin{aligned}
& M_{s_{1}, s_{2}, \eta}^{\alpha_{1}, \alpha_{2}}\left(\left(t_{1}-y_{1}\right)^{2} ; y_{1}, y_{2}\right) \leq \frac{3+\eta(1+\eta)}{s_{1}}=\frac{N_{1}}{s_{1}}, \text { say } \\
& M_{s_{1}, s_{2}, \eta}^{\alpha_{1}, \alpha_{2}}\left(\left(t_{2}-y_{2}\right)^{2} ; y_{1}, y_{2}\right) \leq \frac{3+\eta(1+\eta)}{s_{2}}=\frac{N_{1}}{s_{2}}, \quad \text { say } \\
& M_{s_{1}, s_{2}, \eta}^{\alpha_{1}, \alpha_{2}}\left(\left(t_{1}-y_{1}\right)^{4} ; y_{1}, y_{2}\right) \leq \frac{N_{2}}{s_{1}^{2}} \quad \text { and } \quad M_{s_{1}, s_{2}, \eta}^{\alpha_{1}, \alpha_{2}}\left(\left(t_{2}-y_{2}\right)^{4} ; y_{1}, y_{2}\right) \leq \frac{N_{2}}{s_{2}^{2}}
\end{aligned}
$$

for some constant $N_{1}, N_{2}>0$. Also

$$
\begin{aligned}
M_{s_{1}, s_{2}, \eta}^{\alpha_{1}, \alpha_{2}}\left(\left(t_{1}-y_{1}\right)^{2 m}\left(t_{2}-y_{2}\right)^{2 n} ; y_{1}, y_{2}\right)= & M_{s_{1}, s_{2}, \eta}^{\alpha_{1}, \alpha_{2}}\left(\left(t_{1}-y_{1}\right)^{2 m} ; y_{1}, y_{2}\right) \\
& \times M_{s_{1}, s_{2}, \eta}^{\alpha_{1}, \alpha_{2}}\left(\left(t_{2}-y_{2}\right)^{2 n} ; y_{1}, y_{2}\right)
\end{aligned}
$$

for $\left(t_{1}-y_{1}\right),\left(t_{2}-y_{2}\right) \in I^{2}$ and $m, n=1,2$. From the above and by choosing $\delta_{1}=\sqrt{\frac{1}{s_{1}}}$ and $\delta_{2}=\sqrt{\frac{1}{s_{2}}}$, we have

$$
\begin{aligned}
&\left|\Phi_{s_{1}, s_{2}, \eta}^{\alpha_{1}, \alpha_{2}}\left(h ; y_{1}, y_{2}\right)-h\left(y_{1}, y_{2}\right)\right| \\
& \leq\left\{N_{1} \sqrt{\frac{1}{s_{1} s_{2}}}+2 \sqrt{\frac{N_{1} N_{2}}{s_{1} s_{2}}}+N_{1}^{2} \sqrt{\frac{1}{s_{1} s_{2}}}\right\} \\
& \times \omega_{\text {mixed }}\left(D_{B} h ; \sqrt{\frac{1}{s_{1}}}, \sqrt{\frac{1}{s_{2}}}\right)+3\left\|D_{B} h\right\|_{\infty} N_{1} \sqrt{\frac{1}{s_{1} s_{2}}} \\
&= \frac{1}{\sqrt{s_{1} s_{2}}}\left\{\left(N_{1}+2 \sqrt{N_{1} N_{2}}+N_{1}^{2}\right) \omega_{\text {mixed }}\left(D_{B} h ; \sqrt{\frac{1}{s_{1}}}, \sqrt{\frac{1}{s_{2}}}\right)\right. \\
&\left.+3 N_{1}\left\|D_{B} h\right\|_{\infty}\right\},
\end{aligned}
$$

which yields

$$
\left|\Phi_{s_{1}, \alpha_{2}, \eta}^{\alpha_{1}, \alpha_{2}}\left(h ; y_{1}, y_{2}\right)-h\left(y_{1}, y_{2}\right)\right| \leq \frac{N}{\sqrt{s_{1} s_{2}}}\left\{\omega_{\text {mixed }}\left(D_{B} h ; \sqrt{\frac{1}{s_{1}}}, \sqrt{\frac{1}{s_{2}}}\right)+\left\|D_{B} h\right\|_{\infty}\right\},
$$

where

$$
N=\max \left\{N_{1}+2 \sqrt{N_{1} N_{2}}+N_{1}^{2}, 3 N_{1}\right\}
$$

which completes the proof. 


\section{Acknowledgements}

This work was supported by the Deanship of Scientific Research (DSR), King Abdulaziz University, Jeddah, under grant no. (D-025-130-1438). The author, therefore, gratefully acknowledges the DSR for technical and financial support.

\section{Funding}

This work was supported by the Deanship of Scientific Research (DSR), King Abdulaziz University, Jeddah, under grant no. (D-025-130-1438).

\section{Availability of data and materials}

Not applicable.

\section{Competing interests}

The author declares that he has no competing interests.

\section{Authors' contributions}

The author read and approved the final manuscript.

\section{Publisher's Note}

Springer Nature remains neutral with regard to jurisdictional claims in published maps and institutional affiliations.

Received: 1 October 2020 Accepted: 17 November 2020 Published online: 01 December 2020

\section{References}

1. Acar, T., Aral, A., Mohiuddine, S.A.: On Kantorovich modification of (p,q)-Baskakov operators. J. Inequal. Appl. 2016, 98 (2016)

2. Acar, T., Aral, A., Mohiuddine, S.A.: Approximation by bivariate $(p, q)$-Bernstein-Kantorovich operators. Iran. J. Sci. Technol. Trans. A, Sci. 42, 655-662 (2018)

3. Acar, T., Kajla, A.: Degree of approximation for bivariate generalized Bernstein type operators. Results Math. 73, 79 (2018)

4. Acar, T., Mohiuddine, S.A., Mursaleen, M.: Approximation by $(p, q)$-Baskakov-Durrmeyer-Stancu operators. Complex Anal. Oper. Theory 12, 1453-1468 (2018)

5. Anastassiou, G.A., Gal, S.G.: Approximation Theory: Moduli of Continuity and Global Smoothness Preservation. Birkhäuser, Boston (2000)

6. Aral, A., Erbay, H.: Parametric generalization of Baskakov operators. Math. Commun. 24, 119-131 (2019)

7. Badea, C., Badea, I., Cottin, C., Gonska, H.H.: Notes on the degree of approximation of B-continuous and B-differentiable functions. Approx. Theory Appl. 4, 95-108 (1988)

8. Badea, C., Badea, I., Gonska, H.H.: A test function theorem and approximation by pseudo polynomials. Bull. Aust. Math. Soc. 34, 53-64 (1986)

9. Badea, C., Cottin, C.: Korovkin-type theorems for generalized boolean sum operators, approximation theory (Kecskemét, 1900). In: Colloq. Math. Soc. János Bolyai, vol. 58, pp. 51-68. North-Holland, Amsterdam (1990)

10. Belen, C., Mohiuddine, S.A.: Generalized weighted statistical convergence and application. Appl. Math. Comput. 219, 9821-9826 (2013)

11. Bernstein, S.N.: [Fr]Démonstration du théorème de Weierstrass fondée sur le calcul des probabilités. Commun. Kharkov Math. Soc. 13, 1-2 (1912/1913)

12. Bögel, K.: Mehrdimensionale differentiation von funktionen mehrerer reeller Veränderlichen. J. Reine Angew. Math. 170, 197-217 (1934)

13. Bögel, K.: Über die mehrdimensionale differentiation. Jahresber. Dtsch. Math.-Ver. 65, 45-71 (1962)

14. Braha, N.L., Srivastava, H.M., Mohiuddine, S.A.: A Korovkin's type approximation theorem for periodic functions via the statistical summability of the generalized de la Vallée Poussin mean. Appl. Math. Comput. 228, 162-169 (2014)

15. Cai, Q.-B.: The Bézier variant of Kantorovich type $\lambda$-Bernstein operators. J. Inequal. Appl. 2018, Article ID 90 (2018)

16. Cai, Q.-B., Lian, B.-Y., Zhou, G.: Approximation properties of $\lambda$-Bernstein operators. J. Inequal. Appl. 2018, Article ID 61 (2018)

17. Chen, X., Tan, J., Liu, Z., Xie, J.: Approximation of functions by a new family of generalized Bernstein operators. J. Math. Anal. Appl. 450, 244-261 (2017)

18. Devore, R.A., Lorentz, G.G.: Constructive Approximation. Springer, Berlin (1993)

19. Dobrescu, E., Matei, I.: The approximation by Bernstein type polynomials of bidimensional continuous functions. An. Univ. Timişoara Ser. Şti. Mat.-Fiz. 4, 85-90 (1966)

20. İarslan, H.G.I., Erbay, H., Aral, A.: Kantorovich-type generalization of parametric Baskakov operators. Math. Methods Appl. Sci. 42, 6580-6587 (2019)

21. Kadak, U., Mohiuddine, S.A.: Generalized statistically almost convergence based on the difference operator which includes the $(p, q)$-gamma function and related approximation theorems. Results Math. 73, 9 (2018)

22. Kajla, A., Miclăuş, D.: Blending type approximation by GBS operators of generalized Bernstein-Durrmeyer type. Results Math. 73(1), Article ID 1 (2018)

23. Kajla, A., Mohiuddine, S.A., Alotaibi, A., Goyal, M., Singh, K.K.: Approximation by $\vartheta$-Baskakov-Durrmeyer-type hybrid operators. Iran. J. Sci. Technol. Trans. A, Sci. 44, 1111-1118 (2020)

24. Miclăuş, D.: On the GBS Bernstein-Stancu's type operators. Creative Math. Inform. 22, 73-80 (2013)

25. Mohiuddine, S.A., Acar, T., Alghamdi, M.A.: Genuine modified Bernstein-Durrmeyer operators. J. Inequal. Appl. 2018 104 (2018)

26. Mohiuddine, S.A., Acar, T., Alotaibi, A.: Construction of a new family of Bernstein-Kantorovich operators. Math. Methods Appl. Sci. 40, 7749-7759 (2017) 
27. Mohiuddine, S.A., Ahmad, N., Özger, F., Alotaibi, A., Hazarika, B.: Approximation by the parametric generalization of Baskakov-Kantorovich operators linking with Stancu operators. Iran. J. Sci. Technol. Trans. A, Sci. (2020). https://doi.org/10.1007/s40995-020-01024-w

28. Mohiuddine, S.A., Alamri, B.A.S.: Generalization of equi-statistical convergence via weighted lacunary sequence with associated Korovkin and Voronovskaya type approximation theorems. Rev. R. Acad. Cienc. Exactas Fís. Nat., Ser. A Mat. 113(3), 1955-1973 (2019)

29. Mohiuddine, S.A., Asiri, A., Hazarika, B.: Weighted statistical convergence through difference operator of sequences of fuzzy numbers with application to fuzzy approximation theorems. Int. J. Gen. Syst. 48(5), 492-506 (2019)

30. Mohiuddine, S.A., Hazarika, B., Alghamdi, M.A.: Ideal relatively uniform convergence with Korovkin and Voronovskaya types approximation theorems. Filomat 33(14), 4549-4560 (2019)

31. Mohiuddine, S.A., Kajla, A., Mursaleen, M., Alghamdi, M.A.: Blending type approximation by $\tau$-Baskakov-Durrmeyer type hybrid operators. Adv. Differ. Equ. 2020, 467 (2020)

32. Mohiuddine, S.A., Özger, F.: Approximation of functions by Stancu variant of Bernstein-Kantorovich operators based on shape parameter $\alpha$. Rev. R. Acad. Cienc. Exactas Fís. Nat., Ser. A Mat. 114, 70 (2020)

33. Mursaleen, M., Ansari, K.J., Khan, A.: Approximation by a Kantorovich type q-Bernstein-Stancu operators. Complex Anal. Oper. Theory 11(1), 85-107 (2017)

34. Mursaleen, M., Khan, F., Khan, A.: Approximation properties for modified q-Bernstein-Kantorovich operators. Numer. Funct. Anal. Optim. 36(9), 1178-1197 (2015)

35. Nasiruzzaman, M.: Approximation properties by Szász-Mirakjan operators to bivariate functions via Dunkl analogue. Iran. J. Sci. Technol. Trans. A, Sci. (2020). https://doi.org/10.1007/s40995-020-01018-8

36. Nasiruzzaman, M., Rao, N., Wazir, S., Kumar, R.: Approximation on parametric extension of Baskakov-Durrmeyer operators on weighted spaces. J. Inequal. Appl. 2019, 103 (2019)

37. Özger, F.: Weighted statistical approximation properties of univariate and bivariate $\lambda$-Kantorovich operators. Filomat 33(11), 3473-3486 (2019)

38. Özger, F., Srivastava, H.M., Mohiuddine, S.A.: Approximation of functions by a new class of generalized Bernstein-Schurer operators. Rev. R. Acad. Cienc. Exactas Fís. Nat., Ser. A Mat. 114, 173 (2020)

39. Pop, O.T.: Approximation of B-differentiable functions by GBS operators. An. Univ. Oradea, Fasc. Mat. 14, 15-31 (2007)

40. Rao, N., Nasiruzamman, M.: A generalized Dunkl type modifications of Phillips operators. J. Inequal. Appl. 2018 , 323 (2018)

41. Schurer, F.: Linear positive operators in approximation theory. Math. Inst. Techn. Univ, Delft Report (1962)

42. Srivastava, H.M., Özger, F., Mohiuddine, S.A.: Construction of Stancu-type Bernstein operators based on Bézier bases with shape parameter $\lambda$. Symmetry 11(3), 316 (2019)

43. Volkov, V.I.: On the convergence of sequences of linear positive operators in the space of continuous functions of two variables. Dokl. Akad. Nauk SSSR 115, 17-19 (1957)

44. Wafi, A., Rao, N.: Szász-gamma operators based on Dunkl analogue. Iran. J. Sci. Technol. Trans. A, Sci. 43(1), 213-223 (2019)

45. Wafi, A., Rao, N.: Approximation properties of $(p, q)$-variant of Stancu-Schurer operators. Bol. Soc. Parana. Mat. 37(4), 137-151 (2019)

\section{Submit your manuscript to a SpringerOpen ${ }^{\odot}$ journal and benefit from:}

- Convenient online submission

- Rigorous peer review

- Open access: articles freely available online

- High visibility within the field

- Retaining the copyright to your article

Submit your next manuscript at $\boldsymbol{s p r i n g e r o p e n . c o m ~}$ 